\title{
Bim Expression in Peritumoral Lymphocytes is Associated with Survival in Patients with Metastatic Clear Cell Renal Cell Carcinoma
}

\author{
Bimal Bhindi ${ }^{\mathrm{a}, \mathrm{b}, 1}$, Elizabeth N. Bearrick ${ }^{\mathrm{a}, 1}$, John C. Cheville ${ }^{\mathrm{c}}$, Christine M. Lohse ${ }^{\mathrm{d}}$, \\ Ross J. Mason ${ }^{\mathrm{a}, \mathrm{e}}$, Paras Shah ${ }^{\mathrm{a}}$, Susan Harrington ${ }^{\mathrm{a}}$, Henan Zhang ${ }^{\mathrm{a}}$, Haidong Dong ${ }^{\mathrm{a}}$, \\ Stephen A. Boorjian ${ }^{\mathrm{a}}$, R. Houston Thompson ${ }^{\mathrm{a}}$ and Bradley C. Leibovich ${ }^{\mathrm{a}, *}$ \\ ${ }^{a}$ Department of Urology, Mayo Clinic, Rochester, MN, USA \\ ${ }^{\mathrm{b}}$ Southern Alberta Institute of Urology, Calgary, AB, Canada \\ ${ }^{\mathrm{c}}$ Department of Pathology, Mayo Clinic, Rochester, MN, USA \\ ${ }^{\mathrm{d}}$ Department of Health Sciences Research, Mayo Clinic, Rochester, MN, USA \\ ${ }^{\mathrm{e}}$ Department of Urology, Dalhousie University, Halifax, NS, Canada
}

Received 2 March 2021

Accepted 7 July 2021

Pre-press 26 July 2021

Published 28 August 2021

\begin{abstract}
.
BACKGROUND: Bim (BCL-2-interacting mediator of cell death) is a downstream pro-apoptotic signaling molecule activated by the PD-1 pathway.

OBJECTIVE: We sought to determine if Bim expression in peritumoral T-lymphocytes (PTLs) is associated with survival in patients with metastatic clear cell renal cell carcinoma (ccRCC).

METHODS: Immunohistochemistry staining for Bim was performed on paraffin-embedded tumor tissue blocks from patients with metastatic ccRCC who underwent nephrectomy between 1990-2004. Associations of Bim expression with cancer-specific survival (CSS) and overall survival (OS) from date of metastasis were evaluated using multivariable Cox regression models, adjusting for age, sex, and metastases-score.

RESULTS: 525 patients with metastatic ccRCC, of whom 169 (32\%) had metastases at time of nephrectomy were studied. After multivariable adjustment, high Bim expression remained associated with worse CSS (HR $=1.31$; 95\%CI 1.07-1.59; $\mathrm{p}=0.008)$ and $\mathrm{OS}(\mathrm{HR}=1.28 ; 95 \% \mathrm{CI} 1.06-1.55 ; \mathrm{p}=0.01)$. The interaction between Bim and PD-L1 was not statistically significant for CSS $(p=0.68)$ or OS $(p=0.57)$, suggesting that the associations between Bim and survival outcomes were not significantly different based on tumor PD-L1 expression.
\end{abstract}

\footnotetext{
${ }^{1}$ These authors contributed equally to this work

${ }^{*}$ Correspondence to: Bradley C. Leibovich, MD, Mayo Clinic, 200 1st St. SW, Rochester, MN, 55905, USA. Tel.: +1 507266

0191; E-mail: Leibovich.Bradley@mayo.edu.
} 
CONCLUSION: High Bim expression in PTLs at nephrectomy is prognostic of worse CSS and OS in patients with metastatic ccRCC, irrespective of tumor PD-L1 expression. The role of earlier PD-1/PD-L1-directed therapy warrants evaluation in these patients.

Keywords: Kidney neoplasms, metastasis, nephrectomy, renal cell carcinoma

\section{BACKGROUND}

Approximately $20-30 \%$ of patients with renal cell carcinoma (RCC) present with metastatic disease and 10-20\% of patients who undergo nephrectomy for clinically localized RCC eventually develop metastases [1-3]. The outcomes for these patients are heterogeneous [4]. Notably, there is a subgroup of patients who benefit from a durable cancer-specific survival (CSS) $[4,5]$ and some even experience spontaneous regression of metastases [6,7]. The biological underpinnings of this phenomenal response in the setting of metastatic disease remain to be fully elucidated.

The importance of the interplay between cancer and the immune system has become increasingly evident in recent years. Several malignancies, including RCC, can induce immune tolerance to evade the immune system [8]. It is now recognized that some cancers may exploit the PD-1 pathway to achieve this feat. The PD-1 pathway acts to suppress Tlymphocyte immune response in peripheral tissues to prevent an immune response against autologous cells [9]. PD-1 is expressed on the surface of a number of immune cells, including monocytes, T-lymphocytes, antigen-presenting cells, and tumor-infiltrating lymphocytes. Aberrant expression of PD-L1 by RCC cells can induce T-lymphocyte dysfunction, exhaustion, and neutralization [10].

Bim (BCL-2-interacting mediator of cell death) is a downstream pro-apoptotic signaling molecule in tumor reactive $\mathrm{CD} 8+\mathrm{T}$-cells. Interaction of tumoral PD-L1 with PD-1 on the cell surface of T-cells leads to the upregulation of Bim in tumor-reactive $\mathrm{CD} 8+\mathrm{T}$ cells [11]. In this regard, increased expression of Bim in tumor infiltrating lymphocytes may be indicative of the tumor's ability to evade the immune system. Therefore, in addition to evaluating expression of PD-L1 among tumor cells, it may also be informative to evaluate levels of Bim expression, which may provide a direct demonstration of the PD-1 pathway activation.

To understand the potential role of PD-1 pathway activation in metastatic clear cell RCC (ccRCC) outcomes, we sought to determine if Bim expression in peritumoral lymphocytes (PTLs) is associated with survival in patients with metastatic ccRCC, with the hypothesis that higher Bim expression would be predictive of worse survival.

\section{METHODS}

\section{Study design and participants}

The study was performed in accordance with the Declaration of Helsinki. Ethical approval was provided by Mayo Clinic (IRB\#16-006461).

Following Institutional Review Board approval, the Mayo Clinic Nephrectomy Registry was used to identify 607 patients with ccRCC who underwent radical or partial nephrectomy between 1990 and 2004. Both patients who had metastases at diagnosis (M1) and those who were M0 at diagnosis and subsequently developed metastases were included [4]. Exclusions were receipt of systemic treatments before surgery $(n=5)$ and insufficient tissue available for immunohistochemistry $(n=77)$.

\section{Tumor tissue immunohistochemical (IHC) staining and quantification}

Archival formalin-fixed paraffin-embedded (FFPE) tissue blocks were retrieved from our tissue registry, and tissue sectioning and IHC staining were performed at the Mayo Clinic Pathology Research Core using the Leica Bond RX stainer (Leica). FFPE tissues were sectioned at 5 microns and IHC staining was performed on-line. As we previously reported, slides for Bim stain were run for 20 minutes using Epitope Retrieval 1 (Citrate; Leica) [12]. The Bim primary antibody (Rabbit Monoclonal, Cell Signaling) was diluted to 1:100 in Background Reducing Diluent (Dako) and incubated for 15 minutes.

The detection system used was Polymer Refine Detection System (Leica). This system includes the hydrogen peroxidase block, post primary and polymer reagent, DAB, and Hematoxylin. Immunostaining visualization was achieved by incubating slides 10 minutes in DAB and DAB buffer (1:19 mixture) from the Bond Polymer Refine Detection 
System. To this point, slides were rinsed between steps with 1X Bond Wash Buffer (Leica). Slides were counterstained for five minutes using Schmidt hematoxylin (not provided with Refine kit) and molecular biology grade water (1:1 mixture), followed by several rinses in $1 \mathrm{X}$ Bond wash buffer and distilled water. Once the immunochemistry process was completed, slides were removed from the stainer and rinsed in tap water for five minutes. Slides were dehydrated in increasing concentrations of ethyl alcohol and cleared in three changes of xylene prior to permanent coverslipping in xylene-based medium.

Whole tissue slides were reviewed and quantification of Bim expression was performed by a urologic pathologist (J.C.C.). Bim-positive PTLs were assessed, with the highest number of cells per high power field (hpf) recorded.

\section{Clinical variables, outcomes, and statistical methods}

Features collected at the time of the nephrectomy included year, age, sex, symptoms, Eastern Cooperative Oncology Group (ECOG) performance status, Charlson score, tumor size, the 2018 primary tumor and regional lymph node classifications, presence and level of tumor thrombus, WHO/ISUP grade, coagulative tumor necrosis, and sarcomatoid differentiation. Patients with a palpable flank or abdominal mass, discomfort, gross hematuria, acute onset varicocele, or constitutional symptoms including rash, sweats, weight loss, fatigue, early satiety, and anorexia were considered symptomatic. Features specific to the distant metastases included the timing of metastases in relation to the nephrectomy, metastases arising in the lungs, bone, and liver, multiple sites of metastases, and complete resection of all involved metastatic sites. Additionally, our previously reported Metastases Score, which is based on presence of constitutional symptoms, bone metastases, liver metastases, multiple metastases, tumor thrombus, time from nephrectomy to metastases, whether complete resection of metastatic RCC was performed, grade, and presence of coagulative tumor necrosis was calculated [4].

The co-primary outcomes were CSS and overall survival (OS) as estimated using the Kaplan-Meier method, measured from onset of metastatic disease to death or last follow-up. To avoid immortal time bias, the onset of metastatic disease was considered the date of nephrectomy for patients with metastases at initial diagnosis (M1) and the date of detection of metastases for patients who were non-metastatic (M0) at initial diagnosis.

The clinical and pathologic features studied were summarized with medians and interquartile ranges (IQRs) or frequency counts and percentages. Comparisons of features between groups were performed using Wilcoxon rank sum and chi-square tests. Associations with survival outcomes were evaluated using Cox proportional hazards regression models. All models were adjusted for year of nephrectomy to account for changes in clinical and pathologic features at nephrectomy over time. To understand whether the associations of Bim with survival outcomes were different based on tumor PD-L1 expression, we evaluated the subset of patients with data on both markers, and added PD-L1 expression and an interaction term with Bim to the multivariable models.

Statistical analyses were performed using SAS version 9.4 (SAS Institute, Cary, NC) and R version 3.2.3 (R Foundation for Statistical Computing, Vienna, Austria). All tests were two-sided and $p$-values $<0.05$ were considered statistically significant.

\section{RESULTS}

The final cohort included 525 patients with metastatic ccRCC, of whom 169 (32\%) had metastases at the time of nephrectomy and $356(68 \%)$ of whom were diagnosed with metastatic disease after nephrectomy.

Median peritumoral Bim expression was 20 PTLs/hpf (IQR 3-66; range 0-350; Fig. 1). There was evidence that the association of Bim expression with death from any cause and death from RCC was

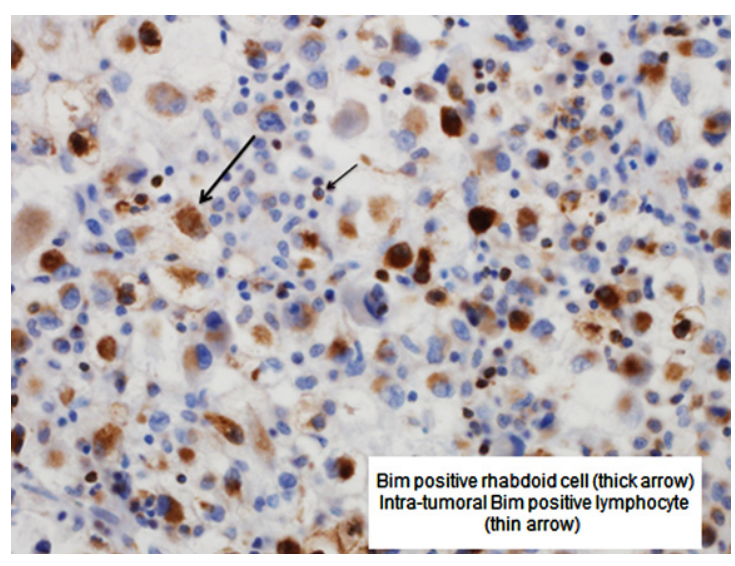

Fig. 1. Bim positive rhabdoid cell (thick arrow) and intra-tumoral Bim positive lymphocyte (thin arrow). 
Table 1

Cohort characteristics

\begin{tabular}{|c|c|c|c|}
\hline Variables & $\begin{array}{c}\text { Low PTL Bim } \\
\text { expression } \\
n=265\end{array}$ & $\begin{array}{l}\text { High PTL Bim } \\
\text { expression } \\
n=260\end{array}$ & $p$-value \\
\hline Continuous variables & Median $(I Q R)$ & Median $(I Q R)$ & \\
\hline Age in years & $61(52-68)$ & $63(55-70)$ & 0.03 \\
\hline Charlson score & $1(0-6)$ & $2(0-6)$ & 0.002 \\
\hline Tumor size in $\mathrm{cm}(n=263: 259)$ & $8.0(6.0-10.5)$ & $10.0(7.2-12.0)$ & $<0.001$ \\
\hline Metastases score & $3(0-5)$ & $5(2-8)$ & $<0.001$ \\
\hline Categorical variables & $n(\%)$ & $n(\%)$ & \\
\hline Female sex & $75(28)$ & $73(28)$ & 0.9 \\
\hline Any symptoms & $183(69)$ & $186(72)$ & 0.5 \\
\hline Constitutional symptoms & $66(25)$ & $95(37)$ & 0.004 \\
\hline \multicolumn{4}{|l|}{ ECOG performance status } \\
\hline 0 & $244(92)$ & $216(83)$ & \multirow[t]{4}{*}{0.001} \\
\hline 1 & $19(7)$ & $33(13)$ & \\
\hline 2 & $1(<1)$ & $8(3)$ & \\
\hline 3 & $1(<1)$ & $3(1)$ & \\
\hline \multicolumn{4}{|l|}{ pT stage $(n=264: 259)$} \\
\hline pT1 & $63(24)$ & $36(14)$ & \multirow[t]{4}{*}{$<0.001$} \\
\hline pT2 & $65(25)$ & $60(23)$ & \\
\hline pT3 & $129(49)$ & $151(58)$ & \\
\hline pT4 & $7(3)$ & $12(5)$ & \\
\hline \multicolumn{4}{|l|}{ Tumor thrombus } \\
\hline None & $185(70)$ & $157(60)$ & \multirow[t]{5}{*}{0.02} \\
\hline Level 0 & $46(17)$ & $50(19)$ & \\
\hline Level 1 & $11(4)$ & $15(6)$ & \\
\hline Level 2 & $10(4)$ & $22(8)$ & \\
\hline Level 3-4 & $13(5)$ & $16(6)$ & \\
\hline pN1 stage & $20(8)$ & $42(16)$ & 0.002 \\
\hline \multicolumn{4}{|l|}{ Grade } \\
\hline 1 & $6(2)$ & $1(<1)$ & \multirow[t]{4}{*}{$<0.001$} \\
\hline 2 & $75(28)$ & $21(8)$ & \\
\hline 3 & $155(58)$ & $171(66)$ & \\
\hline 4 & $29(11)$ & $67(26)$ & \\
\hline Coagulative tumor necrosis & $115(43)$ & $177(68)$ & $<0.001$ \\
\hline Sarcomatoid differentiation & $11(4)$ & $34(13)$ & $<0.001$ \\
\hline \multicolumn{4}{|l|}{ Timing of metastases in relation to nephrectomy } \\
\hline M1 & $75(28)$ & $94(36)$ & \multirow[t]{3}{*}{$<0.001$} \\
\hline $\mathrm{M} 0 \leq 2$ years & $78(29)$ & $99(38)$ & \\
\hline $\mathrm{M} 0>2$ years & $112(42)$ & $67(26)$ & \\
\hline Pulmonary metastases & $140(53)$ & $152(58)$ & 0.2 \\
\hline Bone metastases & $64(24)$ & $55(21)$ & 0.4 \\
\hline Liver metastases & $26(10)$ & $22(8)$ & 0.6 \\
\hline \multicolumn{4}{|l|}{ Number of metastatic sites } \\
\hline 1 & $224(85)$ & $207(80)$ & \multirow[t]{3}{*}{0.2} \\
\hline 2 & $27(10)$ & $44(17)$ & \\
\hline $3+$ & $14(5)$ & $9(3)$ & \\
\hline Complete resection of all metastatic sites & $91(34)$ & $72(28)$ & 0.1 \\
\hline
\end{tabular}

Abbreviations: PTL = peritumoral lymphocytes; $\mathrm{Bim}=\mathrm{BCL}-2$-interacting mediator of cell death $\mathrm{ECOG}=$ Eastern Cooperative Oncology Group.

not linear; as such, Bim expression was categorized at the median for the remaining analyses.

Patients with high (>20/hpf) Bim expression in PTLs were slightly older (median age 63 versus 61 years; $p=0.03$ ), had larger tumors (median $10 \mathrm{~cm}$ versus $8 \mathrm{~cm} ; p<0.001$ ), were more likely to have constitutional symptoms ( $37 \%$ versus $25 \% ; p=0.004)$, had higher pT-stage $(p<0.001)$, were more likely to have pathologic nodal involvement (16\% versus $8 \%$, $p=0.002)$, had higher grade disease $(p<0.001)$, were more likely to have coagulative necrosis $(68 \%$ versus $43 \% ; p<0.001)$ and sarcomatoid differentiation $(13 \%$ versus $4 \% ; p<0.001)$, and had higher Metastases Scores (median 5 versus 3; $p<0.001$ ) compared with patients with low $(\leq 20)$ Bim expression in PTLs (Table 1). 
Table 2

Association between PTL Bim expression and survival outcomes

\begin{tabular}{lcc}
\hline Outcome & $\begin{array}{c}\text { High vs. Low Bim expression HR } \\
(95 \% \text { CI })\end{array}$ & $p$-value \\
\hline Death from RCC & $1.58(1.31-1.92)$ & $<0.001$ \\
$\quad$ Univariable & $1.31(1.08-1.59)$ & 0.006 \\
Adjusted for metastases score & $1.31(1.07-1.59)$ & 0.008 \\
Adjusted for age, sex, metastases score & & $<0.001$ \\
Death from any cause & $1.57(1.30-1.89)$ & 0.004 \\
$\quad$ Univariable & $1.32(1.09-1.59)$ & 0.01 \\
Adjusted for metastases score & $1.28(1.06-1.55)$ & \\
Adjusted for age, sex, metastases score & & \\
\hline
\end{tabular}

Legend: $\mathrm{RCC}=$ renal cell carcinoma; Bim=BCL-2-interacting mediator of cell death; $\mathrm{HR}=$ hazard ratio; $\mathrm{CI}=$ confidence interval.
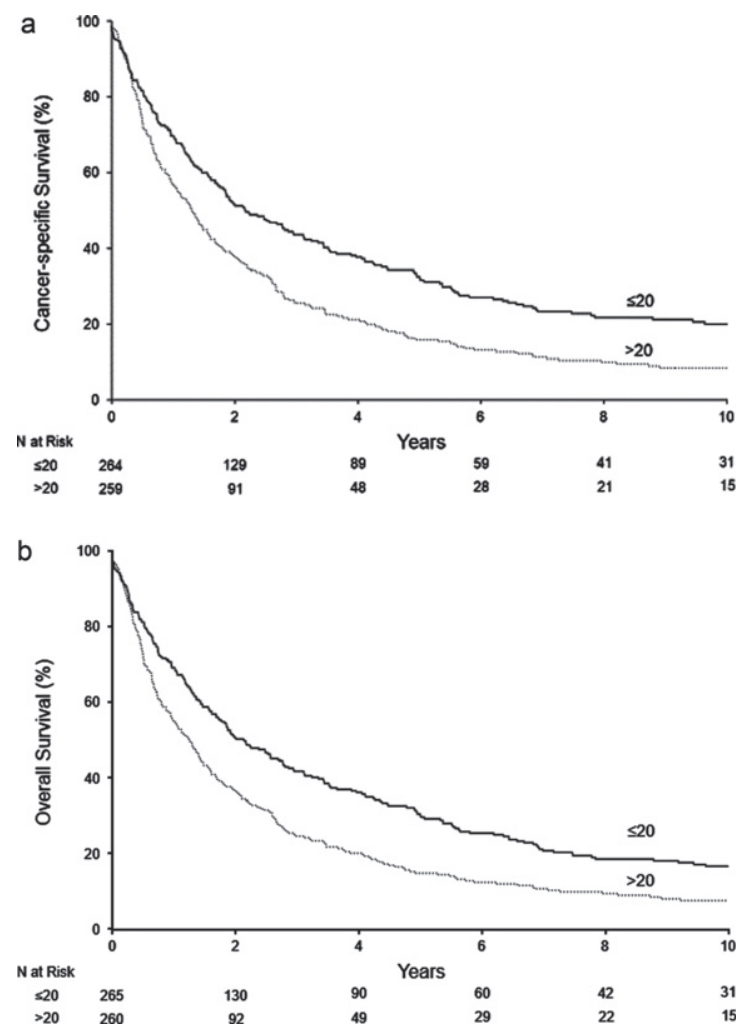

Fig. 2. Survival outcomes according to PTL Bim expression. Abbreviations: $\mathrm{Bim}=\mathrm{BCL}-2$-interacting mediator of cell death.

At a median follow-up of 9.5 years (IQR 4.2-13.1), 468 patients had died, including 434 who died from RCC. Median CSS and OS occurred at 1.7 and 1.6 years, respectively. The survival outcomes of CSS and OS stratified by Bim expression in PTLs are illustrated in Fig. 2. High ( $>20 / \mathrm{hpf}$ ) versus low $(\leq 20 / \mathrm{hpf})$ Bim expression in PTLs was associated with worse CSS $(p<0.001$; Fig. 2a) and worse OS $(p<0.001$; Fig. 2b).
Cox proportional hazards models are shown in Table 2. On univariable analysis, high Bim expression in PTLs was associated with worse CSS $(\mathrm{HR}=1.58 ; 95 \% \mathrm{CI} 1.31-1.92 ; p<0.001)$. After multivariable adjustment for age, sex, and Metastases Score, high Bim expression in PTLs remained significantly associated with worse CSS ( $\mathrm{HR}=1.31$; 95\%CI 1.07-1.59; $p=0.008$ ). Likewise, on univariable analysis, high Bim expression in PTLs was associated with worse OS (HR $=1.57$; 95\% CI 1.30$1.89 ; p<0.001)$. Upon multivariable adjustment for age, sex, and Metastases Score, high Bim expression in PTLs also remained significantly associated with worse CSS $(\mathrm{HR}=1.28 ; 95 \% \mathrm{CI} 1.06-1.55 ; p=0.01)$.

Upon stratifying by tumor PD-L1 (B7-H1) expression among the 453 patients with data on both markers, median Bim expression in PTLs was higher in PD-L1 positive tumors (median $51 \mathrm{PTL} / \mathrm{hpf}$, IQR 22-94) than PD-L1 negative tumors (median 15 PTLs/hpf, IQR 2-60; $p<0.001$; Fig. 3). The interaction between Bim and PD-L1 was not statistically significant for CSS $(p=0.68)$ or OS $(p=0.57)$, suggesting that the associations between Bim and survival outcomes were not significantly different based on tumor PD-L1 expression. The interaction term between Bim and M0 versus M1 classification at nephrectomy was not significant for CSS $(p=0.35)$ or OS $(p=0.36)$, suggesting that the associations between Bim and survival outcomes were not significantly different based on metastatic classification at the time of nephrectomy.

\section{DISCUSSION}

In this study, we found that high Bim expression in PTLs at nephrectomy, a marker of PD-1 pathway activation leading towards apoptosis in tumor-reactive lymphocytes, was associated with adverse pathologic 


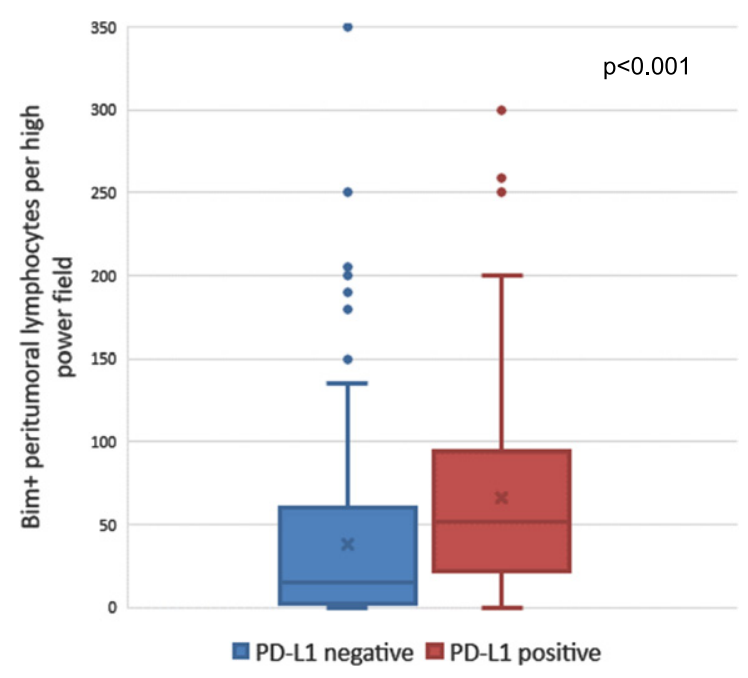

Fig. 3. Number of Bim-positive peritumoral lymphocytes among PD-L1 positive versus PD-L1 negative tumors. Abbreviations: Bim $=$ BCL-2-interacting mediator of cell death; PD-L1 = programmed death ligand-1.

features and significantly associated with worse CSS and OS. As expected, Bim expression in PTLs was higher for PD-L1 positive tumors than PD-L1 negative tumors. However, the interaction between Bim and PD-L1 was not statistically significant for CSS or OS, suggesting that the associations between Bim and survival outcomes were not significantly different based on tumor PD-L1 expression.

PD-1, upon interaction with PD-L1, controls peripheral tolerance by inhibiting T-lymphocyte proliferation, cytokine production, and cytotoxic capabilities, thereby reducing the effectiveness of the T-cell response [13]. PD-L1 expression is not observed in normal kidney tissue and its aberrant expression is associated with both primary and metastatic RCC [14]. Expression of PD-L1 has been associated with more advanced tumor stage, increased tumor size, necrosis, and sarcomatoid differentiation, and worse 5-year CSS [15]. Additionally, increased preoperative PD-L1 levels have been associated with higher tumor stage, grade, and decreased survival [16]. Our data further affirm that inactivating tumor-infiltrating lymphocytes is associated with impaired survival.

While our group is the first to report on the impact of PTL Bim expression in metastatic ccRCC, our results are consistent with other studies in the melanoma literature. Dronca et al. reported that high levels of Bim in circulating tumor-reactive T-cells were prognostic of poor survival in patients with metastatic melanoma who didn't receive anti-PD-1 therapy, which parallels our findings [11]. Since the limitation of IHC in quantitative evaluation of T cell markers, we did not exact know the immuophenotype of Bim in PTL. Given the location of Bim positive PTL and in context of PD-L1 positive tumor cells, we assume the Bim PTLs are likely PD-1 positive cells rather than PD-1 negative cells as we previously reported that PD-L1 only increases Bim expression in PD-1 positive cells [11].

Notably, Bim expression in malignant cells may serve a tumor-suppressor role, which is opposite to the effect of its expression in PTLs [17]. For example, one study found that loss of Bim expression in tumoral cells was associated with reduced apoptosis sensitivity [17]. Meanwhile, Bim expression in tumoral cells has been shown to reduce as melanoma progresses, and reduced Bim expression was found to be significantly associated with poor 5-year survival of melanoma patients [18].

There are several potential clinical applications of Bim that are worth exploring. First, pending further validation, it is possible Bim expression in T-lymphocytes may be a useful prognostic marker in metastatic ccRCC. Second, it will be worthwhile to evaluate whether Bim is a predictive biomarker for response to PD-1/PD-L1-directed therapy. To date, data on tumoral expression of PD-L1 as a predictive biomarker for tumor response to anti-PD-1 therapy have been underwhelming [19, 20]. Given that Bim is a downstream mediator, thus indicating actual PD1 pathway activation, it is possible that Bim may be a better predictive biomarker. Third, it is possible that Bim may be a potential future target for systemic therapy in metastatic ccRCC $[11,21]$. Given recent data supporting the combination of nivolumab, an anti-PD-1 directed therapy, and ipilimumab, an anti-CTLA4 directed therapy, [20] it is possible that combining existing checkpoint inhibitors with an agent targeting Bim may be of value.

There are several limitations worth mentioning. First, we evaluated an older retrospective cohort. While this allows us to better understand how this marker impacts the natural history of metastatic ccRCC and allows for more meaningful follow-up and events, validation in a contemporary cohort is needed. Second, 77 patients did not have sufficient tissue available for IHC staining. However, after multivariable adjustment for age, sex, and Metastases Score, there were no statistically significant differences in CSS $(p=0.7)$ or OS $(p=0.8)$ between those with and without tissue available for analysis. Third, 
we were unable to assess heterogeneity of PTL Bim expression (and tumoral PD-L1 expression) within different areas of the primary tumor, among different metastatic sites, and even among circulating T-lymphocytes. Prospective study of peripheral blood mononuclear cell samples to assess Bim expression in circulating T-lymphocytes in patients with RCC is underway. However, our analysis evaluating the interaction between PTL Bim expression and tumoral PD-L1 expression suggests that the associations between Bim expression and survival outcomes are not significantly different among patients with PD-L1 positive and negative tumors.

\section{CONCLUSION}

Our study found that expression of Bim, a downstream pro-apoptotic mediator of the PD-1 pathway, in PTLs is associated with adverse pathologic features and worse CSS and OS in patients with metastatic ccRCC who have undergone nephrectomy. Further work is needed to characterize the value of Bim expression in PTLs as a prognostic marker and to determine if these patients warrant earlier consideration for novel PD-1/PD-L1 directed agents.

\section{ACKNOWLEDGMENTS}

The authors have no acknowledgments.

\section{FUNDING}

The authors report no funding.

\section{AUTHORS' CONTRIBUTIONS}

All of the named authors have made a substantial contribution to the manuscript and have read and approved the final version.

The authors have had full access to all the data in the study and take responsibility for the integrity of the data and the accuracy of the data analysis.

\section{CONFLICT OF INTEREST}

The authors have no conflict of interest to report.

\section{REFERENCES}

[1] Lam JS, et al. Renal cell carcinoma 2005: new frontiers in staging, prognostication and targeted molecular therapy. J Urol. 2005;173(6):1853-62.
[2] Capitanio U, et al. Epidemiology of Renal Cell Carcinoma. Eur Urol. 2018.

[3] Bhindi B, et al. Are We Using the Best Tumor Size Cutpoints for Renal Cell Carcinoma Staging? Urology. 2017; 109:121-6.

[4] Leibovich BC, et al. A scoring algorithm to predict survival for patients with metastatic clear cell renal cell carcinoma: a stratification tool for prospective clinical trials. J Urol. 2005;174(5):1759-63; discussion 1763.

[5] Alt AL, et al. Survival after complete surgical resection of multiple metastases from renal cell carcinoma. Cancer. 2011;117(13):2873-82.

[6] Marcus SG, et al., Regression of metastatic renal cell carcinoma after cytoreductive nephrectomy. Journal of Urology. 1993;150(2 Pt 1):463-6.

[7] Snow RM, Schellhammer PF. Spontaneous regression of metastatic renal cell carcinoma. Urology. 1982;20(2): 177-81.

[8] Sharma P, et al. Primary, Adaptive, and Acquired Resistance to Cancer Immunotherapy. Cell. 2017;168(4):707-23.

[9] Zitvogel L, Kroemer G. Targeting PD-1/PD-L1 interactions for cancer immunotherapy. Oncoimmunology. 2012;1(8): 1223-5.

[10] Alsaab HO, et al. PD-1 and PD-L1 Checkpoint Signaling Inhibition for Cancer Immunotherapy: Mechanism, Combinations, and Clinical Outcome. Front Pharmacol. 2017; 8:561.

[11] Dronca RS, et al. T cell Bim levels reflect responses to antiPD-1 cancer therapy. JCI Insight. 2016;1(6).

[12] Zhang $\mathrm{H}$, et al. Bim is an independent prognostic marker in intrahepatic cholangiocarcinoma. Hum Pathol. 2018;78: 97-105.

[13] Grzywnowicz M, et al. Programmed death-1 and its ligand are novel immunotolerant molecules expressed on leukemic B cells in chronic lymphocytic leukemia. PLoS One. 2012;7(4):e35178.

[14] Massari F, et al. PD-1 blockade therapy in renal cell carcinoma: current studies and future promises. Cancer Treat Rev. 2015;41(2):114-21.

[15] Thompson RH, Dong H, Kwon ED. Implications of B7$\mathrm{H} 1$ expression in clear cell carcinoma of the kidney for prognostication and therapy. Clin Cancer Res. 2007;13(2 Pt 2):709s-715s.

[16] Frigola X, et al. Identification of a soluble form of B7-H1 that retains immunosuppressive activity and is associated with aggressive renal cell carcinoma. Clin Cancer Res. 2011;17(7):1915-23.

[17] Zantl N, et al. Frequent loss of expression of the proapoptotic protein Bim in renal cell carcinoma: evidence for contribution to apoptosis resistance. Oncogene. 2007; 26(49):7038-48.

[18] Dai DL, et al. Bim expression is reduced in human cutaneous melanomas. J Invest Dermatol. 2008;128(2):403-7.

[19] Motzer RJ, et al. Nivolumab versus Everolimus in Advanced Renal-Cell Carcinoma. N Engl J Med. 2015;373(19): 1803-13.

[20] Motzer RJ, et al. Nivolumab plus Ipilimumab versus Sunitinib in Advanced Renal-Cell Carcinoma. N Engl J Med. 2018;378(14):1277-90.

[21] Akiyama T, Dass CR, Choong PF. Bim-targeted cancer therapy: a link between drug action and underlying molecular changes. Mol Cancer Ther. 2009;8(12):3173-80. 